Report of Meetings, 15,16,17 and 22nd August 1979

PRESTDENT: J. Ring

\title{
Report of the President
}

Since the 16 th General Assembly in Grenoble, an I.A.U. Colloquium (No.50) had been held in Maryland under the auspices of Commission 9 on the subject of "High Angular Resolution Stellar Interferometry". The proceedings of the colloquium had been published - copies were available from Dr J. Davis, Physics Department, University of Sydney, Australia.

Following a suggestion from DrE.H. Richardson and Dr G. Odgers, discussions had begun on the possibility of a Symposium being held at Zelenchuk (the site of the U.S.S.R. 6m telescope) on the subject of "Instrumentation for Large Optical Telescopes". It had proved impossible to organise such a meeting before the 17 th General Assembly but the correspondence would be passed on to the new President.

The President expressed his thanks to the Chairmen of Working Groups who had helped in the production of the "Report of Commission 9". Two mistakes had been pointed out and he took this opportunity of correcting them:

1) In the section on image intensifiers it was reported on page 7 that "On the $6 \mathrm{~m}$ telescope the spectrum of a 16.7 magnitude $s$ tar is recorded in one hour". The exposure was actually six minutes.

2) In the section on television-type systems it was stated that "At Princeton, Lowrance is continuing development of the $70 \mathrm{~mm}$ SEC system..." In fact, this work was discontinued in 1977.

Business Meeting, 15th August

Dr E.H. Richardson was elected President, there being no other nominations.

A ballot was held for the office of Vice-President and membership of the Organising Committee with the following result (the retiring President is customarily included):- Vice President: W.C. Livingstone. Conmittee members:- W. B. Burton; P. Connes; I.M. Kopylov; A. Labeyrie; D. MacMullan (Chairman of Working Group on Detectors); A.B. Meinel; N.N. Mikhel'son; J. Ring (Retiring President); N. Steshenko; G.A.H. Walker; M.F. Walker.

It was agreed after discussion that the Chairmen of Working Groups, who should be elected by the Working Groups, should be co-opted to the Organising Committee. They are as follows:- R. Albrecht (Working Group on Data Processing); J. Davis (Working Group on High Angular Resolution Interferometry); J.L. Heudier (Working Group on Photographic Problems); J. R̈bsch (Working Group on Large Telescope Projects).

Following the decision at the 16 th General Assembly to remove inactive members from the Commission and to encourage new active members, there had been many applications for membership. The list was distributed and, after discussion, all the applicants were elected to membership.

Working Groups

The President pointed out that two of the Working Groups were still only informal ones (Data Processing and High Angular Resolution Interferometry). It was 
agreed that they should henceforth be formally constituted. A discussion ensued on the role and organisation of the Commission's working groups. It was agreed that their functions included:-

(a) The exchange of information between members

(b) The preparation of I.A.U. reports

(c) The organisation of meetings at I.A.U. General Assemblies and elsewhere.

The first function sugges ted that 'membership' of the Working Group (which might in practice mean inclusion in a mailing list) should preferably be open to all members of the I.A.U., whilst the other functions required there to be a nucleus (or Committee). It was agreed that Working Groups should elect their Chairmen and Committee members at General Assemblies and that the composition of the Working Group (or its mailing list) should be left to the discretion of individual groups.

Working Group on Detectors (D. MacMullan) sented.

The group held a meeting on 16 August at which the following papers were pre-

\section{CHARGE COUPLED DEVICES (CCD's)(W. Livings tone)}

Availability and device characterisation are our concern here. We note the Fairchild 201 and 202 ( $100 \times 100$ pixels) are discontinued. The Fairchild 211 $\left(190 \times 244, \frac{1}{2}\right.$ blind, NEC $\left.\sim 20 \mathrm{e}, \mathrm{QE} \sim 12 \%\right)$ and the Fairchild 221 (488x380, I blind, $30(12) \times 18 \mu$ pixels, $Q E \sim 12 \%$ ) are now in use. Fairchild proposes a modified version for astronomy in which the alyminium electrodes are removed. This should increase the QE to about $50 \%$ at $7500 \AA$. No doubt other commercia1 CCD sources of interest to astronomers will soon enter the market.

Because the Fairchild pixel sites are thick $(\sim 250 \mu)$, their cross-section to cosmic rays is high. Marcus, Nelson and Lynds (SPIE vo1. 172, 205 (1979)) report 1 event $/ \mathrm{cm}^{2} / \mathrm{min}$, with each event covering several pixels. Exposure time is thus limited to a few minutes. "Thinning" the array would proportionately reduce this problem.

The above CCD's have no response $\lambda<4000 \AA$. Blue sensitivity may be achieved by thinning (and back illunination) or by over-coating the array with fluorescent dyes (J. Westpha1). Spectral enhancement for $2000<\lambda<4000 \AA$ is obtained with several organic materia1s: Pyrene, Liumogen, and Corronene (W. Be11, KPNO). Ruby is suggested (App1.0ptics 18, 2085 (1979)) but may be impossible to remove in case of deposition error.

Cooling the $\mathrm{CCD}$ to $\mathrm{T}<-100^{\circ} \mathrm{C}$ virtually eliminates pixe1-to-pixel dark current and generally improves uniformity. "Flat fielding" is unnecessary for many applications.

Providing the temperature and control voltages (and timing) are suitably regulated reproducibility to $1 \%$ is realised.

\section{ELECTRONIC IMAGING DEVICES IN SPACE ASTRONOMY (G.R. Carruthers)}

This paper reviews the current and future applications of electronic imaging devices in space astronomy: (1) current flight missions in which electronic imaging devices are the primary astronomical data gatherers, (2) currently approved future missions in which electronic imaging devices will be used, and (3) a sampling of proposed, but not yet approved, future space astronomy investigations of this type.

The current flight missions include two astronomical satellites, the International Ul traviolet Explorer (IUE), and the second High-Energy Astronomical Observatory (HEAO-2, or Einstein). IUE uses intensified $\mathrm{SEC}$ vidicons to record two-dimensional cross-dispersed echelle spectra of $0.1-0.2 \AA$ resolution in the $1150-1950 \AA$ and 1900-3200A wavelength ranges, or low-dispersion (6A resolution) spectra in the same wavelength ranges. Einstein uses a grazing-incidence telescope to image $\mathrm{X}$-rays in the $0.2-4 \mathrm{keV}$ range, with either of two imaging detectors: (a) an Imaging 
Proportional Counter, which has about 1 arc minute angular resolution and $0.5^{\circ}$ field of view, with high detective efficiency, and (b) a High Resolution Imager, based on a chevron microchannel plate with crossed-grid readout, having 3 arc sec resolution but somewhat lower detective efficiency.

Also among current missions are numerous sounding rocket investigations by workers at a number of institutions. Detectors include microchannel image intensifier with phosphor output and film recording (Goddard Space Flight Center), microchannel detectors with resistive anode or discrete anode readouts (University of California/Berkeley, Johns Hopkins University, Naval Research Laboratory, University of Colorado) and electrographic cameras using alkali halide photocathodes (sensitive below $2000 \AA$ ), by NRL.

Approved future investigations include experiments on the Spacelab 1 and Spacelab 2 missions, and the Space Telescope. Spacelab 1 will include the FAUST wide-field all-reflecting camera (Marseilles observatory, France) which uses an MCP intensifier with film recording, and a set of airglow spectrographs covering

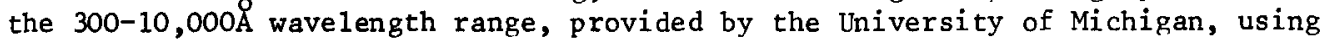
MCP intensifiers coupled to CCD arrays. Spacelab 2 will use a CCD detector as part of the Lockheed solar polarimeter/magnetograph, and microchanne1 plate detector with discrete anode readout as part of an extreme ultraviolet solar spectrograph to be provided by the Appleton Laboratory and University College, London.

The Space Telescope scientific instruments use a variety of imaging detectors. The Wide Field/Planetary Camera (California Institute of Technology) will use $800 \times 800-e$ lement CCD arrays as the image sensors. The ESA-provided Faint Object Cameras will utilize image intensifier/electron-bombarded silicon target camera tubes in photon-counting mode. The Faint object Spectrograph (University of California/San Diego) and the High Resolution Spectrograph (Goddard Space Flight Center) will utilize 512-element linear array (individual readout) Digicon detectors, also in photon-counting mode.

Proposed investigations for future Shuttle/Spacelab missions include both sma11, sounding-rocket-class investigations and larger investigations based on moderate-sized telescopes. In the first class are a high-resolution spectrograph for the 900-1315A range proposed by Princeton University using an electron-bombarded CCD detector, as well as NRL proposals based on electrographic Schmidt cameras (a direct imaging/objective spectrography sky survey experiment, and a nebular spectrograph experiment). Larger instruments include a 0.8 -meter-aperture al1-reflecting Schmidt telescope, covering $5^{\circ}$ fields of view with 1 to 2 arc sec resolution (primary wavelength range 1250-2000A) intended for deep surveys in the far UV, and a Spacelab facility telescope, STARLAB, based on a l-meter aperture Ritchey-Chretien telescope and capable of imaging $0.5^{\circ}$ fields of view with 0.2 arc sec resolution. These latter two instruments would use large-format (120-180 mm image diameter), high-resolution electrographic cameras as the image detectors.

\section{MULTI-ANODE MICROCHANNEL ARRAYS (J.G. Timothy)}

Multi-anode Microchannel Arrays (MAMA's) are a family of photoelectric photoncounting array detectors being developed for use on both ground-based and spaceborne telescopes. The MAMA detector system combines the sensitivity and photometric stability of a conventional photomultiplier tube with a high-resolution imaging capability. MAMA detectors can be operated in a windowless configuration at extreme ultraviolet and soft $X$-ray wavelengths or in a sealed configuration at ultraviolet and visible wavelengths. Prototype MAMA detectors with up to $(512 \times 512)$ pixels are now being evaluated in the laboratory and the first telescope observations with a (10x10)-pixe1 visible-light detector have just been completed. MAMA detectors with $1 \times 512$ pixels, $1 \times 1024$ pixels and $16 \times 1024$ pixels will be operated on the Cassegrain spectrograph of the 24-inch telescope at the University of Colorado during the next twelve months.

\section{ASTRONOMICAL APPLICATIONS OF TELEVISION CAMERA SYSTEMS EMPLOYING ELECTRON BEAM} READOUT (J.Lowrance)

Much of the development work on image sensors and systems for astronomy is now 
on solid state devices. However, the application of more conventional television camera sensors is now a major component in the instrumentation at astronomical observatories throughout the world. Perhaps even more important, these television systems have served to train astronomers in the use of electronic imagery and the data reduction associated with its application to astronomy. This experience makes the adaptation of the solid state sensors to astronomical observations much easier and therefore rapid. In most cases the computer for data acquisition is already available, and the observatory staff is attuned to the task of applving electronic image sensors to astronomical problems. Both Cerro Tololo and the University of Hawaii have developed substantial systems employing silicon vidicons and SIT/EBS tubes controlled by microprocessors or mini-computers as well as the necessary computer equipment and software for data reduction (PASP Vol.91, p.120-139, 1979). Similar equipment is in operation at a few other observatories and it appears that almost all astronomical observatories have some television-based observing system. Most of these systems employ the silicon vidicon, SIT or SEC tubes. (A sumary of the current use of television cameras in astronomical data applications was given by Prof. Lowrance which is not reproduced here.)

CAMERA ELECTRONIQUE (G. W1Erick)

G. Wlérick donne des compléments relatifs a la photométrie par électronographie au télescope de 1.93 mètre de l'Observatoire de Haute-Provence. Toutes les émulsions utilisées en Astronomie son d'abord verifiées en laboratoire par A. Sellier, G. Lelievre et lui-même. Trois propriétés sont examinées: sensibilité, hêtérogéneitié et linearite.

Les Emulsions Ilford G5 et Kodak Industrex (autre nom Definix) son generalment non linéaires pour une densité optique $D>1$, mais elles obéissent à une loi simple a deux parametres comme l'avait vérifie autrefois S. Jeffers (1968):

$$
D=D_{S}\left(1-e^{-A E t}\right)
$$

$\mathrm{D}_{\mathrm{S}}$ densite a saturation, $\mathrm{E}$ éclairement, $t$ temps de pose; pour l'émulsion Industrex, $D_{S}$ varie avec les lots, augmente avec la tension d'acceleration $V$ et vaut $2.5 \mathrm{a}$ 4.5 pour $\mathrm{V}=25 \mathrm{KV}$.

Pour I'emulsion Ilford G5, DS $\sim 4$ pour $V=25 \mathrm{KV}$. L'émulsion L4 est en general linéaire jusqu'a $\mathrm{D}=4$ pour $\mathrm{V}=25 \mathrm{kV}$ c'est à dire que 1a valeur de $D_{\mathrm{S}}$ est très elevée ( $D_{S} \sim 10$ a 20$)$; lorsque 1 a tension d'accéleration diminue $(V \sim 15 \mathrm{KV})$, un Ecart a la linéarité peut apparâtre.

Il est donc essentiel de mesurer les proprietés photométriques des émulsions dans les conditions d'emploi astronomique.

D'autre part, le fait que la densité optique est répresentable par une formule a deux parametres seulement permet d'effectuer facilement les corrections photométriques pour les mesures effectuées avec les microdensitométres tels que le P.D.S.

Working Group on Large Telescopes (Chairman: J. RUsch)

The following papers were presented on August 15 th.

THE INSTRUMENTATION OF THE 6M SOVIET TELESCOPE (I.M. Kopylov)

Here is a short description of the instrumentation now in operation: Prime focus

1. Ritchey cassette. $16 \times 16 \mathrm{~cm}$ plates ( $~ 15 \times 15$ arc-minutes). UBVR colour system. $\mathrm{m}_{1 \text { im }}$ (depending on colour) $23-24.5$.

2. Three-stage image tube device for direct photocathode $\emptyset \approx 40 \mathrm{~mm}$, BVR colour sys tem $\mathrm{m}_{1 \mathrm{~m}} \approx 24-25$.

3. Photometer-polarimeter (two channe1s, ordinary UBVR photometry of faint objects).

3a. Elaborated photometer-computer system aimed at studying very fast brightness variations of optical events around "black holes", neutron stars and intrinsic variable stars. Timescale $10^{3}-10^{-7} \mathrm{sec}$. Time resolution $\simeq \pm 40$ nanoseconds. 4. Two high speed spectrographs with several types of image tubes for spectroscopy 
of faint extragalactic afd stellar objects. Dispersion: $60-270^{\circ} \AA / \mathrm{mm}$, from $3000 \AA$ to near-infrared $(\simeq 9000 \AA)$. m 1 im (depending upon wavelength) up to $19.5-20$. 4a. High speed spectrograph with TV 1000-channel photon-counter scanner controlled and operated by computer and display. $\Delta \lambda 3000-7000 \AA, \mathrm{m}_{1 \mathrm{im}} \simeq 19.5$.

5. TV and image tube devices for the guiding of faint objects on the slit of the spectrograph.

Secondary foci

1. An echelle spectrograph with image tube. Two echelle gratings: 100 and $50 \mathrm{gr} / \mathrm{mm}$, orders $11-20$ and orders 16-25. Dispersion: $30-60 \AA / \mathrm{mm}$ (depends upon grating and wavelength).

2. A spectrograph with high linear resolution (along the slit) with image tube.

3. Three-camera large spectrograph. Two of these cameras are of Schmidt-type. Dispersion (depending up on camera, grating, order, wavelength): $1.3-9-28-56 \AA / \mathrm{mm}$. 3a. Fabry-Perot magnetometer attached to 1 arge camera of P. 3 spectrograph. Controlled by computer. Aimed at the determination of field strength of magnetic stars, $m_{1 i m} \simeq 8$, accuracy of the magnetic field determinations down to \pm 20 gauss. 4. Speckle interferometry device with image tube. $\mathrm{m}_{1 i m} \simeq 8$ (at the moment). Accuracy of determinations of stellar angular diameters: $\simeq \pm 0$ "004. New instrumentation currently being designed and built will be described at the next General Assembly. THE 3.8 METRE UNITED KINGDOM INFRARED TELESCOPE (C.M. Humphries)

The new 3.8 metre U.K. Infrared Telescope (UKIRT) has unique design features in both its structure and its optics. By using a thin primary mirror - very thin by traditional standards - it has been possible to use a relatively lightweight supporting structure and thereby obtain substantial cost savings, a factor of at least three over conventional designs of similar aperture. The CERVIT primary mirror has a mean diameter-to-thickness ratio of only $16: 1$ (compared to 6 : 1 or 8 : 1 for a conventional large mirror) giving a mass of 6 tons instead of 15 tons. It is supported axially by 80 pneumatic pads distributed on three radii and radially by 24 counterweighted lever arms. The telescope has an English yoke mounting and the structure has a mass of 80 tons, instead of 300 or 400 tons. Roller bearings have been used for the polar and declination axes.

The telescope has now been erected at the 4200 metre $(13800 \mathrm{ft})$ site at Mauna Kea, Hawaii, and commissioning is nearly complete. Three focal ratios are available - $f / 9$ Cassegrain, $f / 20$ coude and $f / 35$ Cassegrain with chopped secondary. In each case the secondary mirror acts as an aperture stop and eliminates the possibility of thermal radiation from the tube structure or mirror cell being seen by the detector.

The encircled energy diameters for $90 \%$ of the incident radiation are slightly less than 1 arc sec for the $f / 9$ image and 1.2 arc sec at the $f / 20$ focal plane. The $f / 35$ system is due to be tested within the next few weeks. The optical performance of the telescope has also been obtained in terms of an MTF and compared with measurements of the atmospheric seeing MTF at the site.

At wavelengths above $8 \mathrm{micrometers} \mathrm{the} \mathrm{telescope} \mathrm{is} \mathrm{diffraction-limited} \mathrm{and}$ at all wavelengths the telescope MTF is comparable with or better than the atmospheric MTF for the visible region. The absolute pointing accuracy of the telescope is $\simeq 5$ arc sec RMS with computer corrections applied.

The thin mirror concept has been proved sound in its application to UKIRT and can be used with advantage in other large telescopes planned for the future. This applies equally to equatorial or altazimuth mounting.

\section{THE 3-METER INFRARED TELESCOPE FACILITY (E.E. Beck1in)}

The Infrared Telescope Facility (IRTF) on top of Mauna Kea, Hawaii, became operational during the sumer of 1979. In May, more than twenty-five 5 um images of Jupiter were obtained which were used to help target the Voyager II spacecraft during its July flyby of Jupiter.

The NASA-supported facility is run by the University of Hawaii; it will go into full operation in the Fall of 1979 as a nationally-available facility for infrared research. 
The 3-meter telescope meets a number of criteria which makes it particularly good for infrared observations:

1. The telescope is situated on the summit of Mauna Kea (4120 meters) where there is excellent year-round infrared transmission, lower thermal sky fluctuations and good "seeing".

2. The telescope has an image quality of 0.18 and an image stability of 0.12 ; observations with better than $1^{\prime \prime}$ resolution are possible.

3. The telescope has a very low thermal background and therefore high sensitivity because of the small obscuration in the primary mirror and smal1 undersized secondary mirror. Tests at $10 \mu \mathrm{m}$ show that the telescope can reach $0.005 \mathrm{Jy}$ ( $1 \sigma$ ) in one hour of interpretation.

4. The telescope has versatile $\mathrm{f} / 35$ Cassegrain and $\mathrm{f} / 120$ coude chopping secondaries. The Cassegrain system can efficiently chop an infrared source at $40 \mathrm{~Hz}$ with up to a $5^{\prime}$ throw.

5. The telescope can point very accurately across the sky to better than $5^{\prime \prime}$ under computer control.

LE TELESCOPE CANADA-FRANCE-HAWAII (R. Cayre1, J. Odgers)

Le télescope Canade-France-Hawaii a été monté en usine à La Rochelle-Pallice en octobre 1976 sous abri gonflable pour permettre 1'integration de l'electronique de commande réalisée au Canada et procéder aux essais avant envoi a Hawaii. La rupture du radome fin novembre arrétait les travaux jusqu'en mars 1977 . L'intégration et les essais en usine se sont ensuite poursuivis de mars 1977 a début avri1 1978 et permirent de corriger quelques insuffisances structurales avant le remontage sur le site.

Le télescope a été démonté et emballé d'avril a juillet 1978 et est arrivé a Hawaii le 12 septembre 1978 a bord du navire "Lift Off". Le rémontage sur le site a été effectué de novembre 1978 a juillet 1979.

C'est le 7 août a 12 h 15 TU que la première lumière a été obtenue. Le 11 et 12 aott les premières photographies du ciel etaient prises au foyer primaire.

Le têlescope sera operationnel au foyer primaire dès le debut de 1980 et environ $40 \%$ du temps sera alors attribue aux astronomes visiteurs.

Le foyer coude et le foyer Cassegrain infra-rouge a $\mathrm{f} / 35$ seront mis en service vers la mi-1980. Le foyer Cassegrain $f / 8$ sera mis en service seulement a la fin de 1980 à cause du retard dans le polissage du miroir secondaire.

Une dizaine d'instruments seront progressivement mis en fonction au cours de $1^{\prime}$ année 1980 et au début de 1981 .

LE TELESCOPE DE 2 METRES DU PIC DU MIDI (J. R\&sch, G. Coupinot, G. Carpentier) La construction de ce télescope a eté décidée en 1964 en vue d'exploiter au mieux la qualite des images reconnue au Pic du Midi depuis les travaux de B. Lyot et de des continuateurs. On s'est efforcé de rassembler dans ce projet les solutions permettant d'espérer la meilleure résolution angulaire possible. En particulier, on a procede a une etude en soufflerie de la forme et de la situation du sillage en fonction de la direction du vent, et choisi 1 'emplacement du télescope en tenant compte des vents dominants. On a aussi adopte un type de coupole concue pour éviter tout échange d'air entre l'intêrieur et l'extêrieur, grâce à un joint entre $1^{\prime}$ extrémité du tube et $1^{\prime}$ ouverture circulaire mobile ménagées dans 1 a coupole. On pour:a, par la wuite, fermer 1'avant du tube par une lame a faces paralleles.

La réalisation de $1^{\prime}$ opération, confiée a I'INAG, a débuté en 1970 et a subi de nombreux retards, notamment en raison des intemperies (travaux extérieurs de juin à octobre). La monture, etudiée en même temps que celle du Télescope de $3 \mathrm{~m} 60 \mathrm{CFH}$, est du même type (fer à cheval portant la denture d'entrainement horaire). L'ensemble de la monture et de la coupole est entièrement piloté par un ordinateur, un second pilotant $1^{\prime}$ instrumentation et permettant le traitement des donnés. Le miroir primaire est parabolique, ouvert a $f / 5$. Un Cassegrain $f / 25$ renvoie l'image classiquement par le trou central du primaire; un miroir plan de $1 \mathrm{~m} 10$ de diamètre peut être inséré dans le tube pour renvoyer dans le même plan que le Cassegrain une image a $\mathrm{f} / 5$ (Montage du type Strand). On aprevu la possibilite ulterieure d'un 
foyer coude, et au besoin $d^{8}$ un Cassegrain $f / 15$ et de réducteurs focaux.

L'instrumentation prévue dans une première étape comprend des Caméras Electroniques, une Camera a comptage de photons, un photometre très Elabore, un spectrographe pour objets faibles utilisant des récepteurs $C C D$, et un equipement infrarouge.

On attend la "première lumière" pour la fin de 1979, et on espère que 1'instrument sera operationne1 dans le milieu de 1980.

\section{THE UK NORTHERN OBSERVATORY (G. Smith)}

The UK plans for a Northern Hemisphere Observatory are now materialising at the Spanish "Roque de Los Muchachos" Observatory, on La Palma in the Canary Islands . International agreements on cooperation in astrophysical research, which govern the use of the observatory, were signed between Spain, U.K., Sweden and Denmark on May 26, 1979. Construction of telescope building for Swedish and British telescopes has already begun.

The 1-metre and 2.5-metre telescopes for UK already exist: the 1-metre is approaching completion at Grubb Parsons, and the 2.5 metre is the Isaac Newton, also at Grubb Parsons for installation of a new primary mirror and other improvements.

The designs of the 4.2-metre telescope and its building are almost complete. The alt-azimuth design has led to large economies, both in the telescope mounting and in the reduced dome size. The aim is to have a large, versatile, precision telescope on an excellent site. Versatility is achieved in the alt-animuth design through the availability of the Cassegrain and two Nasmyth foci, with a simple and rapid interchange, as well as the prime focus.

The computer control of the telescope allows stars to be tracked within $1 / 4^{\circ}$ of the zenith. Field rotation will be compensated for in the instrument mountings. Particular attention will be paid in all three telescopes to the control of conditions within the domes, so that full advantage can be taken of the outstandingly good seeing conditions on La Palma.

THE MULTIPLE MIRROR TELESCOPE (D.W. Latham)

(A joint project of the University of Arizona and the Smithsonian Astrophysical Observatory).

On May 9, 1979 the first scientific results from the Multiple Mirror Telescope (MMT) were reported at the MMT Dedication Symposium in Tucson. For these initial applications the six images from the individual 72-inch Cassegrain systems were combined manually. At times of good seeing the individual images appeared smaller than 1 arc second, and the combined image occasionally appeared smaller than 1.5 arc second. The absolute pointing accuracy is already about 5 arc seconds, and undoubtedly this will improve with experience. Even without active correction the images hold together for 10 to 30 minutes, and the tracking is very smooth. Thus many of the aspects of the MMT design, some of them innovative, have already been proven. The six lightweight primaries have been figured accurately to the same radius. The optical support structure, which must carry more than 100 optical elements, performs better than the design goal. The alt-azimuth mount, with its huge azimuth high-angular-contact ball bearing, also works well. The co-rotating building, which has allowed laboratories to be built into the space normally reserved for telescope swing in conventional domes, has proven highly successful. At a total project cost of about 8 million dollars, the MMT falls at least a factor of 2 below the conventional cost curve for a $4.5 \mathrm{~m}$ optical-infrared telescope. The testing and refinement of the active optics are now the main engineering efforts at the telescope, with 5 nights a week scheduled for the engineers and the weekends for the astronomers. We expect this allocation to be $50 / 50$ by the end of 1979 . Until now the scientific applications have used instruments borrowed from other Smithsonian or Arizona telescopes. Major efforts are now going into the development of facility instruments, especially infrared systems and a faint object spectrograph. 
THE NASA SPACE TELESCOPE (N.G. Roman)

The Space Telescope is designed to be the largest and best telescope which is economically feasible. The size, with a $2.4 \mathrm{~m}$ mirror, is the largest which will fit comfortably within the length and weight limit of the Space Shuttle. The guaranteed quality of $0.075 \lambda$ wave-front error for the total system should be achievable with care, an accuracy of $0.05 \lambda$ at $\lambda=6300 \AA$ is hoped for and expected. Even the guaranteed performance will give a resolution of better than 0.1 arc second throughout the visible and ultraviolet. The $\mathrm{f} / 24$ Ritchey-Chretien optics can be used to feed any one of five focal plane instruments. On the first launch, these will be two cameras, two spectrographs and a photopolarimeter. Instruments may be replaced in orbit by new instruments or the entire telescope can be returned to the ground for refurbishment. The cameras cover fields of view from 4 arc seconds to 2.7 arc minutes with angular resolutions increasing from 0.1 arc sec to the highest the optical system can provide in the smallest f.o.v. The spectrographs provide resolutions of $2 \times 10^{4}$ and $1.2 \times 10^{5}$ between 1050 and $3200 \AA$, a resolution of $10^{3}$ between 1050 and $7500 \AA$ and a resolution of $10^{2}$ between 1200 and $7500 \AA$. The photometer will have a time resolution of $16 \mu \mathrm{sec}$ with a spectral resolution up to about $20 \AA$.

\section{NEXT GENERATION TELESCOPE PLANS (D.N.B. Hal1)}

We are presently in a period where a substantial number of ground-based optical and infrared telescopes are being commissioned; a number of these have been described in this session. However there are compelling arguments that astronomers will need very much larger ground-based optical/infrared collecting area in the future. Dramatic improvements in observational capability in other wavelength regimes have invariably required follow-up $0 / I R$ observations, of ten involving long integrations on faint sources; this trend will undoubtedly escalate with the operation of Space Telescope, various infrared satellites, the VLA and large millimeter wave dishes. Yet this explosion in demand will come just when many potential improvements associated with improved efficiency, better detectors and new auxiliary instrumentation will have reached the point of diminishing returns; at this point one can only gain in observing time or limiting magnitude by going to correspondingly larger aperture.

Careful evaluation of the types of astronomical observations for which such a telescope is most suited leads one to the conclusion that the scientific gains come solely as a result of increased area and can be achieved equally well by a single filled aperture or an array of smaller telescopes.

On the other hand, various technical requirements are best met by an array of telescopes in the 5-10 meter size range; these may be independent or attached to a common mount (as in the MMT). With elements smaller than 5 meters, diffraction becomes a factor in image quality in the infrared and the large number of instruments may well be prohibitive. Above 10 meter aperture there are problems matching to foreseeable detectors and spectrographs.

If one scales the cost of conventional large telescopes to a 10 meter aperture using the $D^{2.7}$ power law then costs are prohibitive. However use of thin, possibly segmented, primary mirrors and fast focal ratios hold promise of very significant cost savings and the re are a number of groups in the U.S. now working on designs for telescopes of this class.

\section{Working Group on Photographic Problems (Chairman: J.L. Heudier)}

Two sessions of the Working Group were held during the General Assembly meeting on August 17, 1979. The first session was held jointly with the Working Group on Data Processing, and was attended by about 100 astronomers. The second session, which included the Business Session of the Working Group on Photographic Problems, was attended by about 60 astronomers.

In the joint session, Dr I. King described the responsibilities of the Working Group on Data Processing as including the receipt, reproduction and distribution of materials and information on data processing. Distribution of information is in the form of a newsletter. Requests to be included in the mailing list for the newsletter 
should be addressed to Dr R. Albrecht, Institute for Astronomy, Turkenschanzstrasse 17, Vienna, Austria. Two review papers were presented in this session. In "The Present Status of Astronomical Photography" J.L. Heudier discussed the photographic emulsion as a means of counting photons; the importance of $\mathrm{S}: \mathrm{N}$ considerations, calibration standards, high quality processing, storage and handling requirements, and currently-available techniques of hypersensitization and copying. Dr R. Albrecht discussed "The Measurement and Reduction of Photographic Plates", using as an example the Vienna PDS 1000 system to highlight some of the problems of handling large quantities of information automatically. Attention was drawn to the importance of the communication between such a system and its user - i.e. "user friendliness".

Papers presented in the second session were:- Electronography with the Griboval camera - H.J. Smith and N. Griboval; Calibration - I. Furenlid; UBV Dye Transfer Colour Imagery of Galaxies - J.D. Wray; Hypersensitization of Colour Films - A.G. Smith. It is expected that most of the papers read in these sessions will be published in the AAS Photobulletin.

In the Business Session, Dr R.M. West (Chairman) reported on the activities of the Working Group since the Grenoble meeting. Publications of the Working Group have included the Proceedings of the Grenoble meeting and a Bibliography of Astronomical Photography. A Workshop on Modern Techniques in Astronomical Photography held in Geneva, May 16-18 1978 was well attended, and the Proceedings of that Meeting were published in July 1978. Copies of these Proceedings and of the Working Group publications have been sent throughout the I.A.U. countries to some 400 people on the Working Group mailing list. At a meeting of the Organising Committee held in Geneva it was agreed that the American Astronomical Society Photobulletin should be the recommended journal for publication of papers relating to astronomical photography.

In view of the formal recognition of the Working Group on Data Processing it was necessary to amend the Resolution adopted by the Photographic Working Group during the Grenoble General Assembly, by deleting the clause "and extraction of data from exposed plates".

There are two planned or proposed meetings on astronomical photography. The first, organised by AAS Working Group on Photographic Materials, will be held in San Francisco on January 18 and 19, 1980. There will also be a meeting in Europe early in 1981, probably in Nice.

The membership of the new organising Committee was agreed as follows:Chairman: Dr J.I. Heudier, Observatoire de Nice, B.P. 252, F-06007 Nice-Cedex, France. Secretary: Miss M.E. Sim, Royal Observatory, Blackford Hi11, Edinburgh EH9 3HJ, UK. Members: R.D. Cannon (UK); O. Dokuchaeva (USSR); J. Grygar (Czechoslovakia); D. Latham (USA); D.F. Malin (Australia); A. Millikan (USA); K.R. Sivaraman (India); A.G. Smith (USA); B. Takase (Japan); R.M. West (Denmark). Consultant: W.C. Miller (USA).

A questionnaire concerning the transport of photographic plates had been sent to everyone on the mailing list, resulting in 71 replies from 29 countries. The main problems arise during shipment of the plates from the manufacturer to the customer, when the plates are not under the control of either the supplier of the user. E.J. Hahn and G. Brown of Eastman Kodak reported on their attempts to identify and rectify some of the problems. A Kodak report "Technical Information for Ordering Kodak Spectroscopic Films and Plates" was circulated at the meeting, and will be sent to all Kodak agents and plate users. As a result of internal reorganisation at Eastman Kodak, Mr Gordon Brown has now inherited from Mr E.J. Hahn the responsibility for spectroscopic plates and films, filters and attenuators, and consequently problems which would formerly have been addressed to Mr Hahn should now be addressed to $\mathrm{Mr} \mathrm{Br}$ own.

High Angular Resolution Techniques_ (Chairman: Dr J. Davis)

The following papers were presented on 17th August:- 
A REVIEW OF THE VERY LONG BASELINE AMPLITUDE INTERFEROMETER AT THE UNIVERSITY OF MARYLAND (D. G. Currie)

An amplitude interferometer based on the design of an earlier interferometer, but with two independent telescopes, is being built at the University of Maryland. The interferometer is being set up at a site on the grounds of the optical research facility of the Goddard Space Flight Center in order to carry out a test program. For this purpose the baseline, parallel to the Earth's axis of rotation, has been fixed at $3 \mathrm{~m}$ but the instrument is designed to work in a regular observing program with baselines up to $50 \mathrm{~m}$ at a permanent observing site.

\section{AN 11M PROTOTYPE MODERN MICHELSON STELLAR INTERFEROMETER (J. Davis)}

The Chatterton Astronomy Department of the University of Sydney developed and operated the Stellar Intensity Interferometer at Narrabri for many years and, based on experience gained with that instrument, proposed in 1971 a preliminary design for a very large stellar intensity interferometer. The proposed VLS11 would have a limiting sensitivity $\mathrm{V}(1 \mathrm{imit}) \sim 7.3$ and baselines up to $\sim 2 \mathrm{~km}$. There is no doubt that this instrument could be built and that it would work. However, it would be large, expensive, and the sensitivity would be marginal for some of the most interesting of the programes listed in the introduction to this session.

Although the potential of amplitude interferometry is still to be proven, it is inherently more sensitive than intensity interferometry, and promises to be less expensive. For these reasons the VLS11 has been put on ice while the feasibility of a modern form of Michelson's interferometer is explored by building a prototype instrument based on ideas developed by R.Q. Twiss.

The prototype interferometer will have coelostats to direct light horizontally to a central laboratory from each end of a horizontal 11m North-South baseline. In the interferometer the wavefront distortions induced by the atmosphere will be handled by ( $i$ ) restricting the aperture size to select essentially flat portions of wavefront; (ii) removing wavefront tilts by means of piezo-electrically actuated tilting mirrors; and (iii) removing the effects of randomly varying phase by rapid sampling of the interference and appropriate data handling.

A laser interferometer will be used to monitor internal paths within the interferometer and control a moving retroreflector to equalise the optical paths to the plane of interference.

The design of the interferometer is essentially complete and most of the optical components are in hand. The optical path-length compensation, angular seeing compensation and coelostat guidance servo-systems are being constructed and tested in the laboratory. A potential site is being tested and it is hoped to install and align the instrument during 1980 . The prototype stage is aimed solely at establishing by observations whether fringe visibility can be measured with an accuracy of $\sim 2 \%$ through the atmosphere using $\zeta$ Pup and $\alpha$ CMa as standard reference sources. It is hoped to make these observations during 1981 and to establish optimum instrumental parameters etc. It is intended that the prototype interferometer will ultimately form the heart of a major high resolution instrument.

\section{RECENT RESULTS IN TWO-TELESCOPE INTERFEROMETRY AT CERGA (A. Blazit)}

The small two-telescope interferometer built by Labeyrie has been operational for three years. It consists of two $26 \mathrm{~cm}$ telescopes rolling on tracks on a NorthSouth baseline ranging between 5 and $35 \mathrm{~m}$. The recombination optics and the TV cameras are mounted on a carriage which is moved by a screw controlled by a microcomputer for fringe tracking. Observations are made in white light or in light dispersed by a direct view prism.

Until now, contrasts have been estimated visually, but Laurent Koechlin has built a contrast-measuring device which is being implemented on the interferometer. It measures the contrast of the fringes of each $\mathrm{TV}$ image and a mini-computer gives theix histogram. The histogram is compared with those obtained for reference sequences and the real contrast can be derived with a precision of $5 \%$.

Observational results:-

(i) We have measured 8 stars and observed fringes on 9 non-resolved stars with magnitudes to 3.9 . 
(ii) We are determining a new orbit for Cape11a and our measures of separation now have a precision of $0.4 \times 10^{-3}$ arc. We expect to detect perturbations of this orbit due to a planet or interstellar matter.

\section{A LARGE APERTURE SYNTHESIS ARRAY PROJECT (A. Blazit)}

We have started the construction of a large aperture synthesis array at CERGA. Several telescopes, $1.5 \mathrm{~m}$ diameter, rolling on baselines up to $1 \mathrm{~km}$ will make up this array. Because we need a large number of telescopes free of vibrations and movable on tracks, we have designed a cheap, lightweight telescope which does not need a dome. It consists of a concrete sphere, $3.5 \mathrm{~m}$ diameter, with a cylinder inside. The mount is made of three rings. The optical layout is Cassegrain-Coude with a $1.5 \mathrm{~m}$ primary mirror and a plane tertiary mirror to give a horizontal coude beam. The first telescope is complete and undergoing tests and the second is under construction.

RECENT DEVELOPMENTS OF THE TWO COLOUR REFRACTOMETER TECHNIQUE (D.G. Currie)

For astrometry it is necessary to correct for the atmosphere, otherwise measurements can be little better than current PZT measurements of 0": 15 .

A two colour refractometer has been developed to study the possibility of correction by a dispersion technique. The precision is expected to be between $0 " 1$ and 0":02 for a two-minute observation. The accuracy per observation is expected to be essentially the same, i.e. the systematic or external errors are expected to be less than 0"003 for the PZT.

The method uses a photon-counting quadrant detector system (the automatic guide system or AGS). The AGS has been demonstrated on the 48-inch telescope at the Goddard Optical Research Facility (GORF) to be photon noise-limited to 0"003. Observations are conducted by the use of a rotating wheel with light filters (about $3500 \AA$ and $6500 \AA$ ) which are changed 480 times per second. A prism system nulls the dispersion and the setting of this null position is read as the amount of dispersion.

Test measurements have been conducted and reduced and are photon noise limited (standard deviation of 0.003 in $100 \mathrm{sec}$ ). Initial observations of refraction have been conducted but not analyzed. A full program will be commenced soon.

\section{ASTROPHYSICAL APPLICATION OF SPECKLE INTERFEROMETRY (R. Foy)}

In collaboration with Labeyrie's group, we have carried out two kinds of program using speckle interferometry: (i) resolution of binaries, and (ii) angular diameter measurements.

We observe spectroscopic binaries both to derive stellar masses, and to check stellar evolutionary tracks, by way of composite spectrum binaries. We found that $\zeta^{1}$ Reticuli is a binary; this explains the anomalies of this star. The two components of $X$ Dra have low masses with respect to their temperatures; this could be due to a helium deficiency. We resolved the composite spectrum binary $\delta$ Sge, and confirmed that the orbit is highly inclined; an atmospheric eclipse should occur next spring (1980).

The discrepancy, by a factor of ten, about the gravity of Arcturus, raised by Malckle et al (1975) has been explained by Martin (1977). Giving a large weight to the angular diameter neasurement of Arcturus by Blazit et al (1977), she proposed to return to the higher value of the gravity and to a mass of 1 solar mass.

Speckle observations can be used to probe the atmospheres of Mira Variables we found that the angular diameter of Mira and $R$ Leo are markedly larger in the strong bands of Titanjum oxide than outside. The difference reaches a factor of two between 7000 and $7100 \AA$. Using model atmospheres by Tsuji, we showed that this difference is due to the very large opacity coefficient of Tio: the optical depth in TiO bands is unity far above the stellar surface.

We noted an increase of the diameter of Mira during maximum of November ' 78 with respect to maximum of December '76, which implies that Mira was cooler in November '78. This agrees with the fact that Mira was then fainter by 1.5 magnitudes than in December' 76. 
IMAGE RECONSTRUCTION WORK AT THE AIR FORCE GEOPHYSICS LABORATORY (S.P. Worden)

Several aspects of astronomical image reconstruction work are being pursued at the Air Force Geophysics Laboratory. In collaboration with contractors at Harvard the application of speckle interferometry to studies of the solar surface is being investigated. The use of an active optics unit developed by ITEK for similar high resolution solar observations is also being studied. In concert with groups at the University of Arizona and Lockheed, systems have been developed to observe faint objects and, in some cases, completely reconstruct images using speckle interferometry techniques. Efforts to adapt the Center for Astrophysics/Arizona Multiple Mirror Telescope for speckle imaging work have also begun.

\section{INTERFEROMETRY FROM SPACE (A. B1azit)}

Ground-based interferometers have already provided results on stars at visible wavelengths, but for extragalactic objects atmospheric fluctuations will limit the integration time and prevent the observation of objects of magnitude fainter than 15 , such as quasars and nuclei of Seyfert galaxies. A space interferometer would have enough angular resolution and sensitivity to observe faint extragalactic objects in the visible and UV. It will also give dimensions and perhaps mapping of stars and systems of stars over a large range of wavelengths. The most important objects are: Stellar objectives: Extension of layers of cool supergiants/hot stars; Star formation; Structures in molecular clouds; Structures in the discs of stars; Close binaries with transfer. Extragalactic objectives: Active regions in nuclei; Shocks in dusty regions of nearby galaxies; Quesars, Seyfert nuclei. Astrometry: Absolute references; Obscure companions.

The design we have selected for the space interferometer is the "flute" tube. Two telescopes, Im in diameter, move inside a structural tube with a certain number of viewing holes. Several sections of $15 \mathrm{~m}$ could be assembled together in orbit. Extensions to $100 \mathrm{~m}$ or more appear feasible.

The infrared range could be covered with this instrument if the central package of optics and detectors is made interchangeable. Observation of faint objects will require offset guiding on the fringes of a bright star. It could be realised with 3 telescope carriages, and a triple interferometer. Three fields could be observed simultaneously $120^{\circ}$ apart on a great circle of the celestial sphere. Two guide stars could be used for absolute stabilisation, while observing a faint object. This project has been submitted to E.S.A.

A SPACE APPLICATION OF THE VERY LONG BASELINE AMPLITUDE INTERFEROMETER (D.G.Currie)

Certain of the scientific and technical apsects of the operation of a Very Long Baseline Amplitude Interferometer (VLBAI) (similar to the VLBAI currently being developed by the University of Maryland for ground-based operations) were discussed.

The Space-based VLBAI (SVLBAI) will initially provide an astrophysical capability to observe and resolve much fainter objects than may be observed from the ground. Of particular interest are the cores of QSO's, BL Lac objects, Seyfert galaxies, and the inner core of "conventional" galaxies. It should also provide an astrometric capability to study fainter stars, with higher accuracy, than may be done from the ground. This would include parallax studies, the search for extrasolar planets and a study of the relation between the FK4 catalog and the QSO/BL Lac "inertial" frame of reference.

In the first instance a ten metre system (i.e. a few metres separation between the apertures) is being considered. This, will serve as a prototype for later expansion to thirty and one hundred metres. Each of the end mirrors would consist of four segments, each about $0.75 \mathrm{~m}$ diameter, which are independently moveable. Since these need point only with "diffraction" accuracy, not "interferometric" accuracy, the existing Automatic Guiding System provides the requisite pointing accuracy. The object acquisition is done with a specially modified, thinned CCD. The interferometric delay is maintained with the AGS for the low frequency and a gyro system for the high frequency. The measurement of the differential delay between two stars is done with the fringe counting laser system. A system with a graphite inner truss 
and a heat-pipe/mu1ti-layer insulation outer structure guarantees sufficient insulation from the thermal effects of the sun.

The expected precision in a planetary search program or a study of the para1lax of fainter stars ( 15 th magnitude) would be 0.005 milliseconds of arc for a 1000 second integration and a 100 metre system. For the QSO studies, the precision would be 1 millisecond of arc for a 25 th magnitude object for the same conditions. A preliminary study of the accuracy questions indicates that the obvious systematic errors are not incompatible with the above examples of precision.

Working Group on Infrared and General Techniques (Chairman: J. Ring) (22 August)

The following papers were given:

EARLY OBSERVATIONS WITH THE UK INFRARED TELESCOPE AND ITS INSTRUMENTS (T.J. Lee)

Commissioning work on instruments and some observations for astronomical research programmes have taken place during the later stages of commissioning. Some instrumentation was described together with its performance. Examples of observations made were shown. Some of the astronomy to be done 1ater in 1979 was outlined.

A CASSEGRAIN, SCANNING FABRY-PEROT INTERFEROMETER WITH AN IMAGING DETECTOR

(P. Atherton, D.J. Axon \& K. Taylor)

TAURUS, a new Fabry-Perot imaging device, designed to obtain complete seeinglimited radial velocity field maps of extended emission-line sources, was described. A servo-controlled Fabry-Perot is used with a focal reducer and a 2-dimensional photon-counting (area detector) system, to obtain the velocity information. The system has been fully tested and the first observations of the highly complex velocity field of the irregular galaxy M82 were used to illustrate the power of the ins trument.

A CASSEGRAIN, FABRY-PEROT/ECHELLE SPECTROMETER (B. Bates)

An outline was given of a Fabry-Perot echelle spectrograph to be used for first observations in Autum 1979. The instrument is designed to record a large number of spectral elements simultaneously at a resolving power $\approx 3 \times 10^{5}$. The interferometer is combined with an echelle order sorter to provide a compact spectral format suitable for image intensifier recording. The technique is based on that employed successfully during a programme of UV interference spectroscopy from a moderately stabilized balloon-borne platform.

\section{AN INTERFEROMETRIC STELIAR-OSCILLATION SPECTROMETER (A. Forrest \& J. Ring)}

The usefulness of accurate measurements of the radial velocities of stars was discussed, together with the limitations to the accuracy of such measurements imposed by the properties of current instruments. Two new interferometric methods were described; the first employed a Michelson interferometer preceded by a narrow-band interference filter centred on a stellar absorption line. The path-difference in the interferometer was fixed at about $5 \mathrm{~mm}$ and servo-controlled with the aid of a stable laser. The flux of starlight emerging from the two outputs was monitored changes in the difference in intensity of the two beams was a measure of a change in the radial velocity of the stellar atmosphere and was used to detect stellar oscillations. The instrument had been tested on the Sun and the 300 s oscillation had been detected with an accuracy of a few metres per second in the measurement of the radial velocity amplitude.

The second method involved the use of a solid Fabry-Perot interferometer, the thickness of the plate being controlled by temperature using a laser as a wavelength reference. The wavelength of a stellar absorption line should be measured to an accuracy which would allow radial velocity changes of a few metres per second to be detected. This instrument was still under construction. 


\section{POSSIBLE ADVANTAGES OF AN ALT-ALT TELESCOPE SYSTEM FOR STELLAR SPECTROSCOPY}

(T. Dunham Jr)

Large and heavy equipment for analyzing light from stars and planets, particularly high resolution spectrographs and systems for Fourier spectroscopy, must be carried on a rigid foundation. This requires a telescope that brings light to a fixed focus. Ordinarily, an asymmetrical equatorial coude mounting is employed for this purpose. But this design requires heavy castings and expensive machine work to provide adequate rigidity. It also requires four mirror reflection.

An alt-alt mounting (AURA Engineering Technical Report No.38, KPNO 1971 and A. Labeyrie, Ap.J. Letters, L71, 1975) involves a simpler mechanical yoke design. Cost should be considerably less, since construction does not require special machinery. And only three reflections are required to deliver light to a fixed focus.

The two axles of an alt-alt telescope must be driven at continuously changing rates by a computer which sends pulses to stepping motors, or controls the rates of D.C. motors. Such computer controls have recently been used with success for large alt-azimuth telescope mountings. Cost could be moderate if encoder feedback is not included, and if an observer provides visual-manual guiding. Performance should be better than with most large telescopes ten years ago.

For small telescopes, the computer drive for an alt-alt telescope will make the total cost for the instrument more than for an equatorial coude system. But, since the cost of a computer drive is almost independent of the aperture of the telescope, an alt-alt mounting should be less expensive than an equatorial coude telescope for large apertures. It seems likely that costs for the two designs may be approximately equal for an aperture of about $80 \mathrm{~cm}$. If $\mathrm{so}$, then the cost, in terms of photons delivered per second per dollar invested, should be approximately equal for telescopes of about $70 \mathrm{~cm}$ aperture, because the alt-alt system requires one less reflection.

An alt-alt mounting is being seriously considered for a 50-inch telescope to feed 1ight to a stationary stellar spectrograph in Australia. Comments and suggestions from members of Commission 9 will be welcomed.

\section{SECOND-ORDER STATISTICS FOR IMAGE CLASSIFICATION (M.L. Malagnini)}

A new procedure was described for image classification using a computer-controlled scanner, designed in particular for the identification of globular clusters in M31. The approach is based on the analysis of second-order statistics of the grey-level distributions of the images of different categories of object. Classification parameters are derived from a test-set of known objects. Results were presented for a few hundred objects which showed the potentialities of the method. 